\title{
Mechanical Properties Improvement of Polyester Composite Reinforced with Sisal Fibers Treated by Different Methods
}

\author{
Luju He $^{1, \text { a }}$, Li Ma ${ }^{2, b,{ }^{*}}$, Xianyi Shao ${ }^{3, c}$ \\ ${ }^{1}$ Education and Information Technology Center, Guangdong University of Petrochemical \\ Technology, Maoming 525000, Guangdong, China \\ ${ }^{2}$ College of Mechanical and Electrical Engineering, Guangdong University of Petrochemical \\ Technology, Maoming 525000, Guangdong, China \\ ${ }^{3}$ College of Physics \& Electronic Engineering, Taizhou University, Taizhou 318000, China \\ aheluju@gdupt.edu.cn, ${ }^{b}$ mali@gdupt.edu.cn, ${ }^{c}$ sxy8718@163.com
}

Keywords: Sisal fibers; Tensile strength; Surface treatment; Composites

Abstract. Elevated environmental awareness and an increasing concern with the global warming have stimulated the automotive, construction and packing industries to look for materials that can replace conventional synthetic fibers. Natural fiber appears as a good alternative since they are available in fibrous form and can be extracted from plant leaves, stalk, fruits and seeds at very low costs. In this study, the effect of sisal fiber surface treatment on mechanical properties was studied for natural sisal fibers. Sisal fibers were treated by alkali treatment, heat treatment and silane treatment respectively in order to enhance the interfacial adhesion of the fiber/polymer composite for mechanical properties improvement. Sisal fiber/polyethylene (PE) composites were prepared at fiber content of 10, 20, 30wt\% by using twin screw extrude equipment and their mechanical properties such as tensile, flexural and impact strength were examined. Silane treated fiber (with content of $30 \%$ )reinforced composites showed 18\% increase in tensile strength and 32\% in Young's modulus, while the alkali treated fiber (with content of 30\%)reinforced composites performed $37 \%$ increase in flexural strength. However, in case of impact strength, the treatment has been found to cause a reduction. It was also found with increasing fiber content, tensile strength, flexural strength and modulus of the PP composites increased but impact strength and elongation at break decreased.

\section{Introduction}

The composite materials in polymer matrix reinforced with natural fibers are receiving more attention in both academia and various industries. The mechanical properties of a natural fiber-polymer matrix composite are controlled to a large extent by the efficiency of the bonding at the fiber-matrix interfacial boundary. The principal function of the interface is to facilitate the transfer of stress from fiber to fiber, across the matrix. Cellulose fiber is known to exhibit poor bonding characteristics with a polymeric matrix ${ }^{[1]}$. This is mainly due to the presence of organic and inorganic substances on the fiber surface, and the hydrophilic nature of cellulose, which prevents effective adhesion between the fiber surface and the polymer matrix. The surface of natural fiber such as sisal fiber is usually not suitable for creating a strong bond with a polymeric matrix. The bond strength has been successfully improved by modifying the fiber surface with either chemical or physical surface treatment such as heat treatment ${ }^{[2]}$, corona treatment ${ }^{[3]}$, plasma treatment ${ }^{[4]}$, silanization ${ }^{[5]}$ and acetylation ${ }^{[6]}$.

In this work, the effect of interfacial modification including alkalization, heat treatment and silane treatment on mechanical properties of sisal/PP composite was studied, and the effect of fiber content on properties of composite was examined, in an attempt to produce high strength and stiff PP composites.

\section{Experiment details}

Materials. The industrial sisal fibers were supplied by China Oriental sisal Group Company. The commercial grade of Polypropylene used as matrix was supplied by Mao-ming Polypropylene Co., 
Ltd. Trimethoxy-silane was purchased from Nanjing Chen industrial organic silicon material Co., Ltd. All other chemical used were of analytical grade obtained from local commercial sources.

Surface treatment. Pre-dried fibers were handled with alkali treatment (referred as AL), heat treatment (referred as HT) and silane treatment (referred as SL), respectively. They were soaked in $6 \%$ sodium hydroxide solution at room temperature for 1 hour. After treatment, fibers were washed to remove alkali on the fiber surface. Heat treatment was performed by heating untreated sisal fibers in an oven at $400 \mathrm{~K}$ for an hour under atmospheric pressure. A solution of $1.0 \mathrm{wt} \%$ silane coupling agent was prepared in acetone. Untreated sisal fibers were immersed in the solution for 2 hour, and then they were removed from the solution and dried in oven at $400 \mathrm{~K}$ for 12 hours.

Composite preparation. Chopped dried short untreated (referred as UF) or treated sisal fibers and PP pellets were compounded with fiber content of 10, 20,30wt\% by using ThermoPrism twin screw extruder for good mixing of fiber and polymer. The extruded composite material was palletized and dried at $350 \mathrm{~K}$ for 48 hours and then injection molded using an injection molding machine.

Characterization methods. Tensile properties of PP and PP composites were tested on a Zwick machine at room temperature, with a load cell of $250 \mathrm{kN}$, following normative ASTM D3039. The impact testing was carried out according to ASTM D256 using an impact testing machine. Composite fracture surface morphology was studied with HITACHI S-4700 field emission scanning electron microscopes. All samples were sputter-coated with platinum and palladium to provide enhanced conductivity.

\section{Results and discussion}

Tensile properties. Fig.1. shows the typical stress-strain records of the PP and composite. It can be seen that the curves initially show linear deformation, followed by a small non-linear deformation prior to the attainment of the maximum strength. The average Young's modulus, tensile strength and elongation at break of the untreated and treated sisal fiber/PP composites are shown in Fig.2, 3, 4. We can observe that Young's modulus and tensile strength to be increased after every modification. Among all the samples, Young's modulus and tensile strength of the PP/SL composites with fiber content of $30 \%$ were to be the highest of which reached $1.25 \mathrm{GPa}$ and $30.06 \mathrm{MPa}$, respectively, about $32 \%$ and $18 \%$ higher than those of the PP/UF composites. Silane is a multifunctional molecule which is used as a coupling agent to modify fiber surfaces.

The composition of silane forms a chemical link between the fiber surface and the matrix through a siloxane bridge. Silanols form in the presence of moisture and hydrolysable alkoxy groups in condensation process, one end of silanol reacts with the cellulose hydroxyl group and the other end reacts with the matrix functional group. This co-reactivity provides molecular continuity across the interface of the composite. It can also provide hydrocarbon chain that restrains fiber swelling into the matrix ${ }^{[11]}$. As a result, adhesion between sisal fiber and matrix has been improved. Heat treatment technique can bring an enhancement in fiber-matrix interfacial adhesion which was due to removal of impurities, hemicelluloses and lignin on fiber surface resulted in improved compatibility between the fiber and the polymer matrix. The effect of alkali treatment is in agreement with the reported works which have been published on jute, hemp and kenaf ${ }^{[7-8] .}$ It has been found that alkali was the most effective method in treating the sisal fiber and enhancing the interfacial adhesion properties. The alkali treatment acts on the middle lamella and on the primary cell wall resulting in removing the hemicelluloses and dissolving pectin. With increasing fiber content, Young's modulus and tensile strength of the PP composites with or without interfacial modification were increased as shown in Fig.2 and 3, respectively. However, elongation at break of PP composites decreased as displayed in Fig.4. The higher amount of fibers increased a probability of fiber agglomeration and created void in the composites. The silane treatment of fibers also increases the tensile strength and modulus of the composite. The SEM of silane treated fiber (Fig. 5(d)) shows the presence of macrospores, rougher fiber surface and fibrillation. This could be attributed to good adhesion in between treated fibers and PP as illustrated decreased fiber pull-out in Fig.5 (a) which represents the micrograph of the untreated date sisal fiber. We can see that there is a layer covered the fibers named waxy layer which led to 
worsen the interfacial adhesion of the fiber with the matrix.

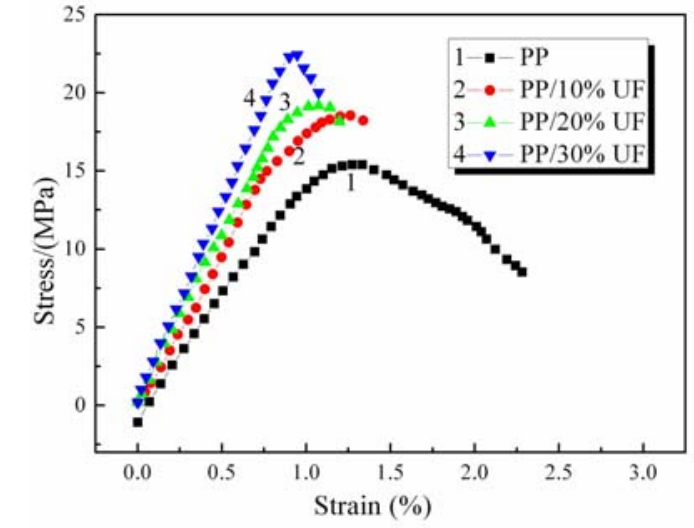

Fig.1. Stress-strain curves for tensile testing of PP and composites

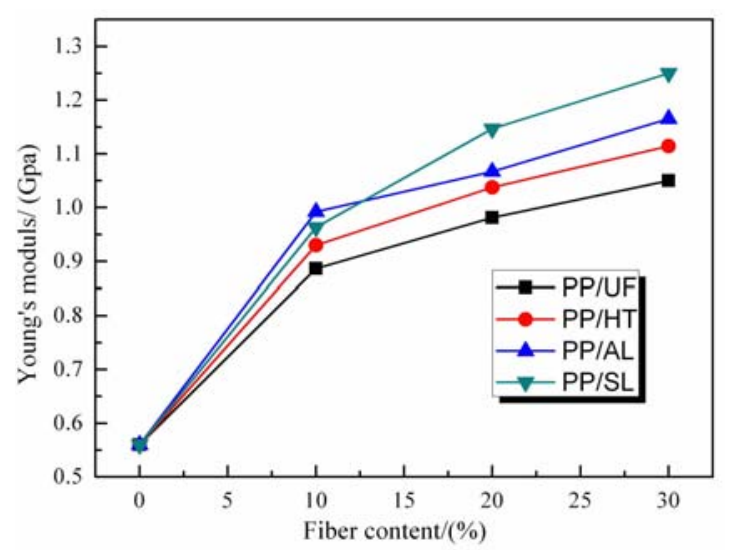

Fig.3. Young's modulus of various treated sisal fiber/PP composites with different fiber content
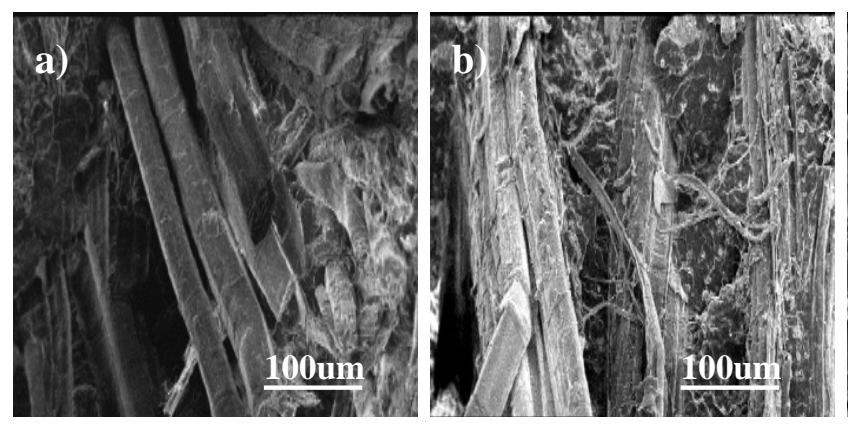

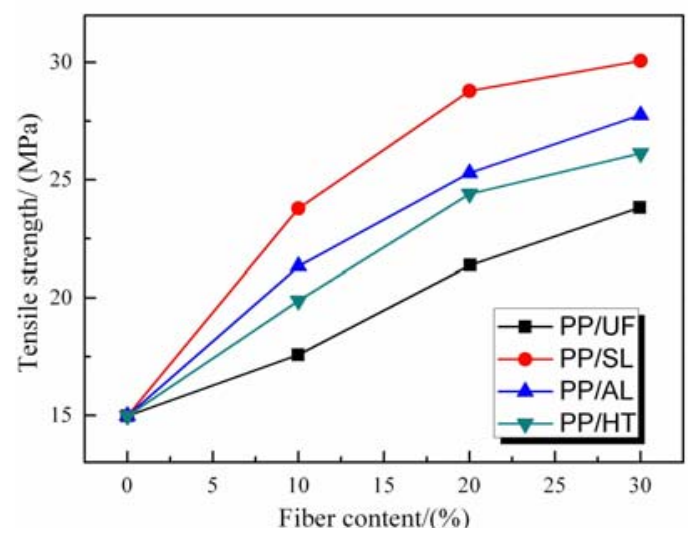

Fig.2. Tensile strength of various treated sisal fiber/PP composites with different fiber content

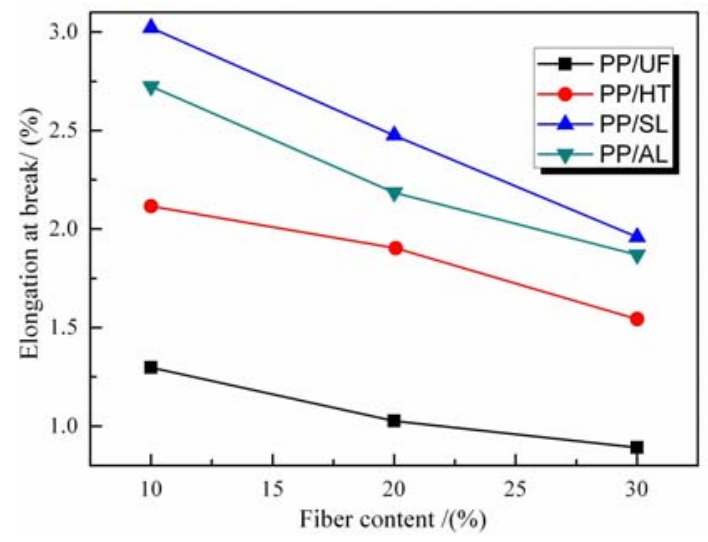

Fig.4. Elongation of various treated sisal fiber/PP composites with different fiber content
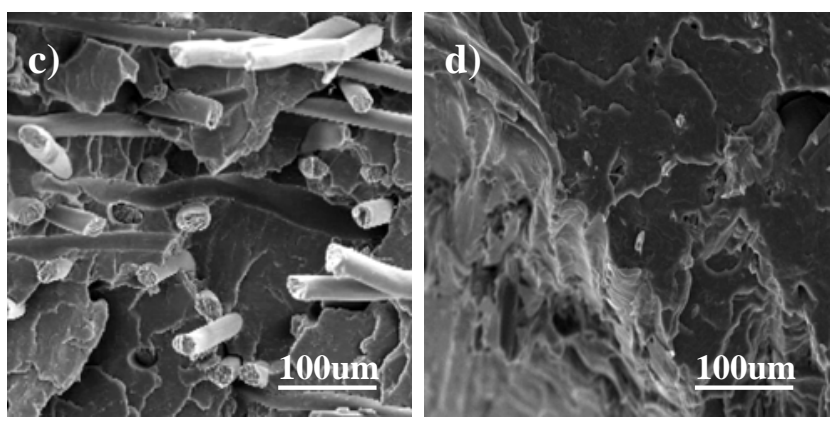

Fig.5. SEM micrographs of tensile fracture surface of untreated and treated sisal fiber reinforced composites: (a) PP/UF; (b) PP/HT; (c) PP/AL; (d) PP/SL

Impact tests. The variation of impact strength of untreated and treated sisal fiber reinforced PP composites was depicted in Fig.6. It is clear that impact strength of all composites increased with increased fiber content. It is because as sisal fiber content increased, more interfaces exist on the crack path and more energy would be consumed. The concentration of fibers would have increased with increased fiber content which lead to increase pull-out and also increased impact strength. It can be seen from Fig.6 that the impact strength of SL, AL and HT composites is lower than that of UF composites. The lowest value is seen for the SL composites with highest Young's modulus and tensile strength. Improved interaction will result in perfect bonding and thus the failure of the composites could occur at relatively low impact. Ray found that in composites having weak interfacial bonding the crack propagated along the fiber-matrix interface caused debonding which lead to a significant increase in the energy absorbing capacity of the composites. As a result, new surfaces produced and 
the frictional work resulting from differential displacement between sisal fibers and matrix increased the impact fatigue resistance of the composites.

Flexural tests. The effect of treatments in relation to the interface bonding characteristics between fiber and matrix is reflected on the flexural strength properties. Fracture behavior and flexural strength properties of untreated and treated sisal enforced PP composite are shown in Fig.7. It can be seen that the flexural strains decreases drastically for the treated fiber reinforced composites. The flexural properties of treated and untreated sisal fiber reinforced polyester composites are presented follow the order: AL $>$ SL $>\mathrm{HT}>\mathrm{UF}$. After treatment the alkali treated fiber reinforced composites performed a maximal 37\% increase, while the silane treated and heat treated fiber showed a maximal $26 \%$ and $35 \%$ increase in the flexural strength respectively.

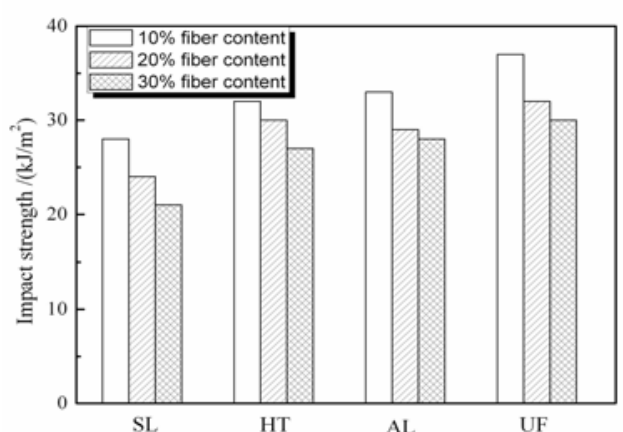

Fig.6. Impact strength of various treated sisal fiber/PP composites with different fiber content

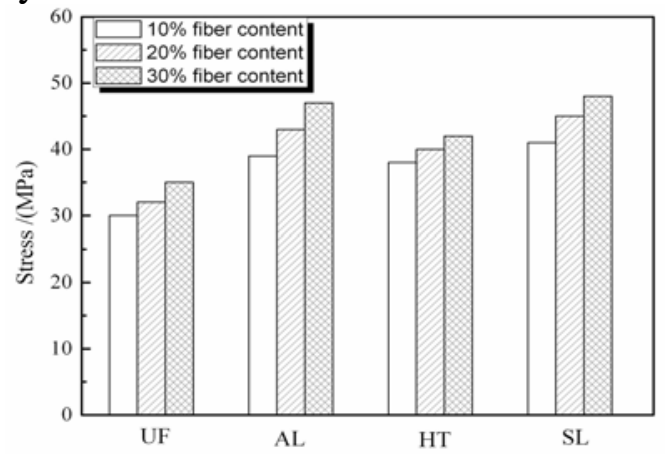

Fig.7. Flexural strength of various treated sisal fiber/PP contents with different fiber content

\section{Summary}

The sisal fiber has been treated with different interfacial treatments and sisal fiber/PP composites with different sisal fiber content were fabricated by screw extrudes equipment. The test results of tensile and flexural show that mechanical properties increase after every treatment due to improved interfacial interactions. Silane treatment provided the most effective enhancement in tensile properties of the PP composites, and alkali treatment provided the most effective enhancement in flexural properties of the PP composites, however, the impact strength of composites after interfacial treatment is lower than that of untreated sisal fiber reinforced PP composites.

\section{Acknowledgements}

The authors are grateful for the financial support of this research from Maoming Science \& Technology Project (No. 915325), Talent Recruitment Foundation from Guangdong University of Petrochemical Technology (No. 650119) and Taizhou Science \& Technology Project (No. 14GY02).

\section{References}

[1] A. Ramzy, D. Beermann, L. Steuernagel, D. Meiners: Composites Part B Vol. 66(2014), p.287

[2] G.A. Holt, P. Chow, J.D. Wanjura: Industrial Crops and Products Vol. 52(2014), p. 627

[3] A. Pizzi, R. Kueny, F. Lecoanet, et.al: Industrial Crops and Products Vol.30 (2009), p. 235

[4] R.H. Bradley, I.L. Clackson, D.E. Sykes: Applied Surface Science Vol. 72(1993), p. 143

[5] D. García-García, A. Carbonell, M.D. Samper : Composites Part B Vol. 78(2015), p.256

[6] I. Ben Amor, M. Arous, A. Kallel: Journal of Electrostatics Vol. 72(2014), p. 156

[7] M.M. Kabir, H. Wang, K.T. Lau, F. Cardona: Composites Part B Vol. 53(2013), p.362

[8] O.M.L. Asumani, R.G. Reid, R. Paskaramoorthy: Composites Part A Vol. 43(2012), p.1431 